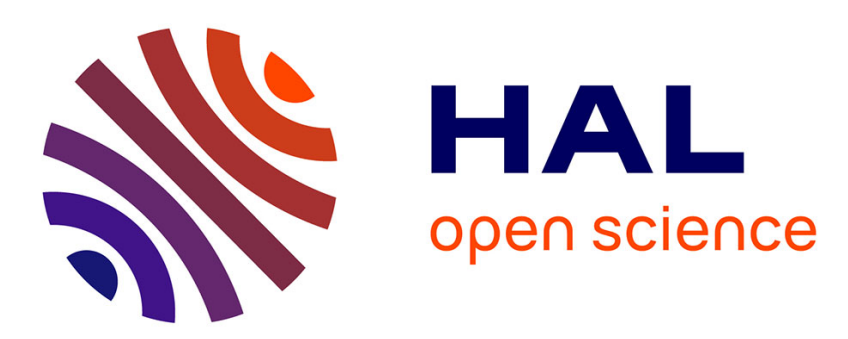

\title{
Adaptable dialogue architecture and runtime engine (AdaRTE): A framework for rapid prototyping of health dialog systems
}

\author{
Lina Maria Rojas Barahona, Toni Giorgino
}

\section{- To cite this version:}

Lina Maria Rojas Barahona, Toni Giorgino. Adaptable dialogue architecture and runtime engine (AdaRTE): A framework for rapid prototyping of health dialog systems. International Journal of Medical Informatics, 2009, MedInfo 2007 special issue, 78 (Supplement 1), pp.S56 - S68. 10.1016/j.ijmedinf.2008.07.017 . inria-00519747

\section{HAL Id: inria-00519747 \\ https://hal.inria.fr/inria-00519747}

Submitted on 21 Sep 2010

HAL is a multi-disciplinary open access archive for the deposit and dissemination of scientific research documents, whether they are published or not. The documents may come from teaching and research institutions in France or abroad, or from public or private research centers.
L'archive ouverte pluridisciplinaire HAL, est destinée au dépôt et à la diffusion de documents scientifiques de niveau recherche, publiés ou non, émanant des établissements d'enseignement et de recherche français ou étrangers, des laboratoires publics ou privés. 


\title{
Adaptable Dialogue Architecture and Runtime Engine (AdaRTE): A Framework for Rapid Prototyping of Health Dialogue Systems
}

\author{
L. M. Rojas-Barahona, T. Giorgino \\ Laboratory for Biomedical Informatics, \\ Dipartimento di Informatica e Sistemistica, \\ Università di Pavia, Italy.
}

\begin{abstract}
Spoken dialogue systems have been increasingly employed to provide ubiquitous access via telephone to information and services to the non-Internet-connected public. They have been successfully applied in the health care context; however, speech technology requires a considerable development investment. The advent of VoiceXML reduced the proliferation of incompatible dialogue formalisms, at the expense of adding even more complexity. This paper introduces a novel architecture for dialogue representation and interpretation, AdaRTE, which allows developers to layout dialogue interactions through a high level formalism, offering both declarative and procedural features. AdaRTE's aim is to provide a ground for deploying complex and adaptable dialogues whilst allowing experimentation and incremental adoption of innovative speech technologies. It enhances Augmented Transition Networks with dynamic behavior, and drives multiple back-end realizers, including VoiceXML. It has been especially targeted to the health care context, because of the great scale and the need for reducing the barrier for a widespread adoption of dialogue systems.
\end{abstract}

Keywords: Telemedicine, Speech Recognition Software, Ambulatory Care Information Systems, Telephone, Computerized.

\section{Introduction}

Automated dialog systems are widely used to provide the public with access to a set of automated services. The opportunities offered by computer-based conversations can be reaped for telemedicine applications, e.g. offering patients a self-paced access to an ever-increasing fraction of clinical information. Patients can take the role of either information consumers (e.g. for receiving counsel and 
education), producers (interviews, long-term monitoring of chronic diseases, symptom reporting, etc.), or both (e.g. as a support to the scheduling of clinical exams and receiving therapy updates).

Adopting dialogue systems in the medical domain, however, is especially complex. First, dialogues in health care context should be designed to maintain a continuous relation with patients through the time. Some dialogues have the objective of eliciting changes in patient's behaviours or habits. Criticality is also present in dialogues used for chronic symptoms monitoring [1]. Last but not least, clinical practices enact complex guidelines, ontologies and procedures [2], which increment the complexity of automated dialogues. A number of these clinical practices in patients' home care require professional assistance to be successfully fulfilled. At the same time, it is simply unfeasible to have the whole medical personnel available to cover patients' demands and, perhaps, this is the main motivation towards the adoption of automatic dialogue systems in medicine. Another important motivation is to increase availability of costeffective monitoring in disadvantaged geographical regions.

This paper presents the AdaRTE framework, devised in order to overcome the issues of the existing dialogue management methods. Our interest is mainly focused on health care dialogues systems, and therefore our solution is especially targeted at offering rapid prototyping, standards-compliant deployment and experimentation through the incremental integration of other voice formalisms, e.g. NLP based on lexicalized grammars.

AdaRTE's features were designed to reduce the burden of dialogue development through reuse, support of augmented transition networks, adaptable decision points and adoption of best practices. This paper shows how AdaRTE implements these features for dialogue deployment, and presents the results obtained prototyping five medical telephony-linked systems. Three of them were inspired by earlier working systems, namely the Chronic Obstructive Pulmonary Disease (COPD) care [3], the Homey dialogue system for hypertensive patient home management [4], and for diabetes care [5]. Two more dialogues were implemented from scratch to assist patients receiving peritoneal dialysis and Oral Anticoagulation Therapy (OAT); the latter is being prepared for use at the Mondino Neurological Hospital of Pavia (Italy). 


\section{Background}

Computer-based dialogue systems have been proven useful to provide the general public with access to telemedicine services. Several studies have discussed their advantages for chronic symptoms monitoring, interviews, counselling and education. Piette, Corkrey, Krishna, Kaplan and Edmonds [6-10] reviewed interactive voice response systems (IVR) that have been used for interviews, alcohol or drug-abuse support, hypertension monitoring, and others, emphasizing the benefits of these systems such as the ability to be in continuous operation whilst offering confidentiality. Migneault [11] summarizes the experience in building over twenty IVR systems for various health purposes focusing on positively influencing patient's health behaviour and disease monitoring such as angina pectoris, chronic obstructive pulmonary disease, asthma and others. Several interventions have been reportedly able to improve quality of service and communication in a cost-effective way. Also, dialogues supporting automated speech recognition (ASR) have been used successfully for health purposes. For instance, a dialogue for the management of hypertensive patients supporting high adaptability and restricted mixed initiative is described in [4]. More recently, Levin presented a system for chronic pain monitoring, tested on volunteers, that profiles users upon their experience, offering varying amounts of help, and fallbacks in case of misunderstanding by the ASR component [12]. Further discussion on the adoption of dialog systems for health communication can be found in a special issue [13].

Despite the growing efforts towards developing user-friendly IVR systems, this kind of telemedicine interventions have been criticized for their lack of flexibility, mainly due to the fact that, to be effective, they greatly restrict the interaction with the user. This is mostly due to technical reasons: touch-tone based systems are limited to numeric input and menu-like navigation. On the other hand, speech-based system emerged as a very promising solution thanks to the extensive efforts pursued by the speech recognition community. Nevertheless, speech-based systems are still error-prone and their language understanding component doesn't cover the whole variety of linguistic phenomena [14]. 


\subsection{Theoretical approaches}

Attempts towards more sophisticated approaches to dialogue modelling in the medical domain have been pursued in $[15,16]$. In the former, Allen et al. present a medication advisor "Chester", in which the dialogue is seen as a collaborative system where agents work together in order to achieve a common goal. Chester embraces the generic architecture developed for The Rochester Interactive Planning System (TRIPS) [17], which clearly separates domain and dialogue representations. It uses a stochastic plan and an intention recognizer in order to infer the user's intention and supports mixed initiative. In the latter, Beveridge et al. present a decision support dialogue system for advising physicians about whether or not a patient should be referred to a cancer specialist. The dialogue follows the conversational games theory of dialogue modelling, first introduced by Power [18]. Its architecture separates dialogue and domain representation and, in particular, the domain and plan representation use ontologies and guidelines, respectively.

In spite of the remarkable endeavours, a considerable effort should still be done in order to solve issues related to language, pronunciation modeling of medical terminology, limited information in knowledge bases, computational costs for reasoning and adaptability. This is even more evident when considering the perspective of implementing robust, functional and complete dialogue systems for real situations in the medical domain.

A range of approaches is available for dialogue modeling. According to the classification made by Allen et al. [19] ordered by increasing complexity, the simplest of these is finite-state scripts, also called dialogue grammar, followed by frame-based, sets of context, plan-based, and agent-based models. In a finite-state script the dialogue is represented as a script of prompts for the user. In frame-based systems, questions are asked in order to enable the system to fill slots of entity requirements to perform a task. Sets of contexts describe the dialogue task and each context is represented using the frame-based technique. Conversely, plan-based theories claim that utterances infer acts that are part of a plan, thus, the system should identify the user underlying plan and respond appropriately [20]. Agent-based models are at the highest level of complexity. They consider planning, executing and monitoring operations in a dynamically changing world, possibly involving multimodality. An additional approach, the 
conversational games theory, is presented in $[16,18,21]$. This approach models task-oriented dialogues and uses techniques from both frame-based and plan-based models. This approach provides a method of modeling mixed-initiative and complex dialogues.

Generally, many of the aforesaid approaches require heavily coded solutions and are not readily suited for small-scale applications in a real-world setting. Additionally, there is not much information available about time and costs implied in the dialogue systems development process. As a matter of fact, the process of deployment dialogue systems has been considered complicated, costly, time demanding and usually requires speech technology experts. Several toolkits were devised in order to simplify the programming burden; among them, the best-known is probably TrindiKit [22]. Although TrindiKit allows the implementation of dialogues following the theoretical approach of information state, it is complex to use and requires the proprietary language SICStus Prolog.

\subsection{Standardization of technology}

Recently, the WWW Consortium introduced the web-based "Voice Browser" (VB) activity [23]. VBs are built around a dialogue manager, which fetches documents over the web and interprets them. Its delivery reduced the proliferation of incompatible dialogue formalisms by offering a reference model for voice applications. Dialogue systems built with VoiceXML have been published lately, e.g. for home monitoring of diabetic patients [24]. In spite of the aforesaid advantages, VoiceXML has inherent limitations which are well analyzed in $[25,26]$. For instance, its structure is declarative, static and it lacks means for efficient and heavy computation, so it is difficult to access remote resources (e.g. databases and ontologies). Also, its support to mixed-initiative interaction is limited. Furthermore, due to the web-based paradigm, VoiceXML documents themselves have to be generated dynamically by other code, which complicates application maintenance. Finally, the strongest limit pointed out by the research community is that neither dynamic natural language understanding, generation nor multimodality are directly supported. 


\section{Motivation}

Early methods for describing the detailed structure of computerized voice interactions followed either the finite-state or the frame-based approach. Technically, they were implemented either in native computer code, or with custom dialog managers. In the former, the interaction was specified and hard-coded in a custom program, step by step, in a native computer programming language, for example C. Such a program activates speech and telephony-related features on demand according to the point of the dialog, by means of platform-specific application-program interfaces (API) which depend on the particular hardware and software combination. This approach is familiar to computer programmers and allows fine control over all aspects of the interaction. However, it ties implementations to the specific software or hardware vendors, is not portable, and puts the dialog flow design mainly in the hands of programmers, rather than domain experts.

\subsection{Context-based approaches}

The fact that most of the telephone interaction boils down to a few steps of basic types, e.g. playing prompts, listening to answers, storing results in variables, and so forth, has motivated dialog engines to be built around context-based approaches [27, 28]. The definition of "context" differed among platforms, but usually it was characterized by a group of properties, such as: prompt, grammars, variables to hold the result, help, and pointer to the next context.

Although this formalism simplifies dialog layout by decomposing it into basic building blocks, and provides a higher level of abstraction than low-level computer languages, it still has the disadvantages of being proprietary and strongly tied to the specific dialog manager. Furthermore, it generally lacks the flexibility required for handling special, application-specific cases - e.g. to compute prompts adaptively on the basis of user experience or other factors, to enable or disable confirmation questions, and generally it's difficult to implement strategies beyond those supplied with the platform as built-ins. These refinements tend to be important for a smooth user experience, but often can be expressed readily only through procedural programming (e.g. to keep track of counters and sensible defaults, perform non-trivial 
string analysis, hold complex states in an object-oriented fashion, and so on). Procedural and objectoriented constructs conflict with the static declarative structure of context-based dialog formalisms.

\subsection{Conforming to industry standards}

VoiceXML has been a first step towards a combined procedural and declarative approach, because it has foreseen both a form-filling mechanism and a procedural interpreter, in the form of the standard ECMAScript standard language [29]; context variables and scripts belong to the same namespace. In addition, ECMAScript is a powerful general-purpose language, but its usefulness is severely limited by being confined on the client-side. Therefore, this interesting approach is limited by the web-based paradigm, because the document generation and exchange have to be meta-programmed and executed at runtime, and the procedural interpreter is restricted to the browser. Both the high-level dialog management and the resources access take place on the web server, where they have to be programmed with the less-than-straightforward mechanisms like Java Server Pages. Again, this paradigm may be familiar to programmers, but is often outside of the grasp of domain experts for dialog writing or maintenance. For the reasons discussed in the previous section, VoiceXML has a negative impact on the ability to produce telemedicine applications efficiently.

As a consequence, a variety of extensions to VoiceXML have been proposed: for instance, DialogXML (applied to car telematics) extended the VB in order to support NLP KANTOO generated grammars [30]. A prototype of an editor for creating VoiceXML documents is exposed in [31]. Other VoiceXML generative approaches are presented in [32], which follows a database-oriented approach, and in [33], which is seemingly targeted towards customer care tasks with sophisticated call routing. We believe that a big effort should still be done in adapting dialog systems best practices [34], such as confirmation strategies, adaptability, mixed initiative into VoiceXML-based frameworks, providing usable speech interfaces to users and graphical interfaces to developers.

We devised a dialog representation that overcomes these limits. This work has been motivated by two main factors: (1) reduce time required to deploy dialogue applications, and enable subjects who were not 
programmers to develop and test autonomously their applications; and (2) experiment with best practices and alternative dialog strategies, incorporating them in existing systems, where possible, without rewrite. (So-called "best practices" suggest consistent adoption of dialog features like self-revealing prompts, incremental amounts of help, smart recovery from ASR recognition errors, and so on. [34])

\section{Adaptable Runtime Engine}

The approach we propose is AdaRTE, a flexible dialog manager patterned around the combined procedural-declarative approach to dialogue-based interfaces. According to Allen's classification [19], the new approach falls in the "sets-of-context" category, because it combines the static nature of contexts and slots with the dynamic features of a procedural interpreter. The interpreter provides enough flexibility in execution in order to switch contexts according to arbitrarily complex criteria. The main components of the proposed architecture are (a) dialogue interpreter, (b) a runtime engine and (c) an interface media realizer for back-end generation (Figure 1). A system typically interacts with three main roles: dialogue developers, patients, and case managers (e.g. physicians or case manager nurses, typically through a web interface).

The flow of a conversation is structured as a series of Augmented Transition Networks (ATN). Usually, an ATN is associated with a context or a topic; we call this structure subdialogue. Subdialogues partition a complex application into modules (Figure 2). This contributes to structure the conversation layout for ease of maintenance, because subdialogues can be reused both within the same dialog, and across different applications (Figure 3).

Prompts, questions and other elements are the nodes (here named blocks) of an ATN that specifies the flow of the conversation. Blocks are represented in the description by XML tags. When the system is started, the XML dialogue description is read and compiled into an internal representation. When a call reaches the system, the dialog representation is executed. Consequently, AdaRTE activates the dialogue blocks, constructs prompts, interprets the answers returned by the caller through the voice platform, and interacts with external resources as appropriate. 
When a call is setup, the main subdialogue is retrieved and started; it will, in turn, invoke other subdialogues, and so forth. When the end of each subdialogue is reached, the execution flows returns to the caller, and at the end of the main subdialogue, the call is terminated. Subdialogues flow can be altered by throwing dialog-specific exceptions.

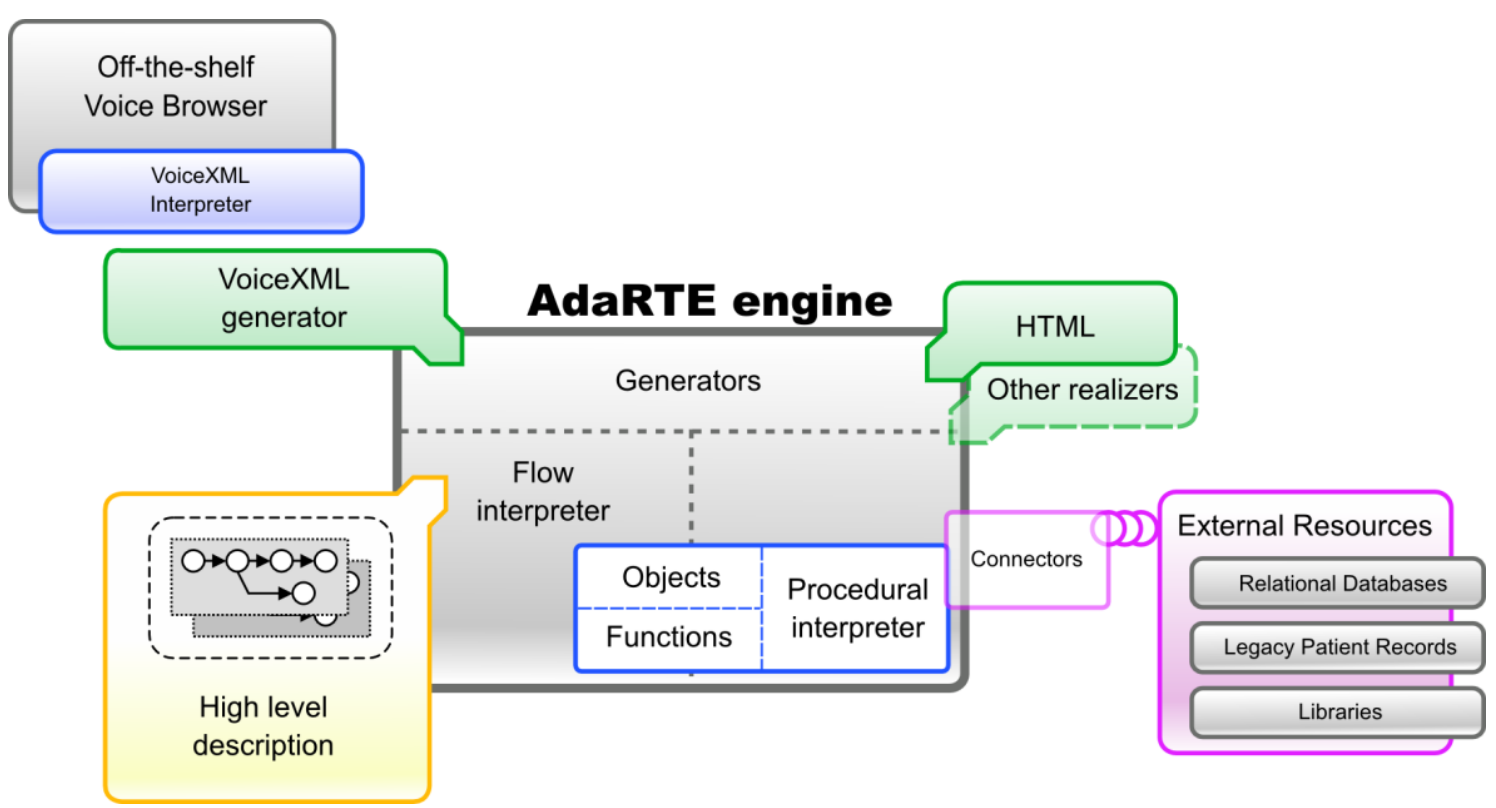

Figure 1 Block diagram of the AdaRTE architecture. 


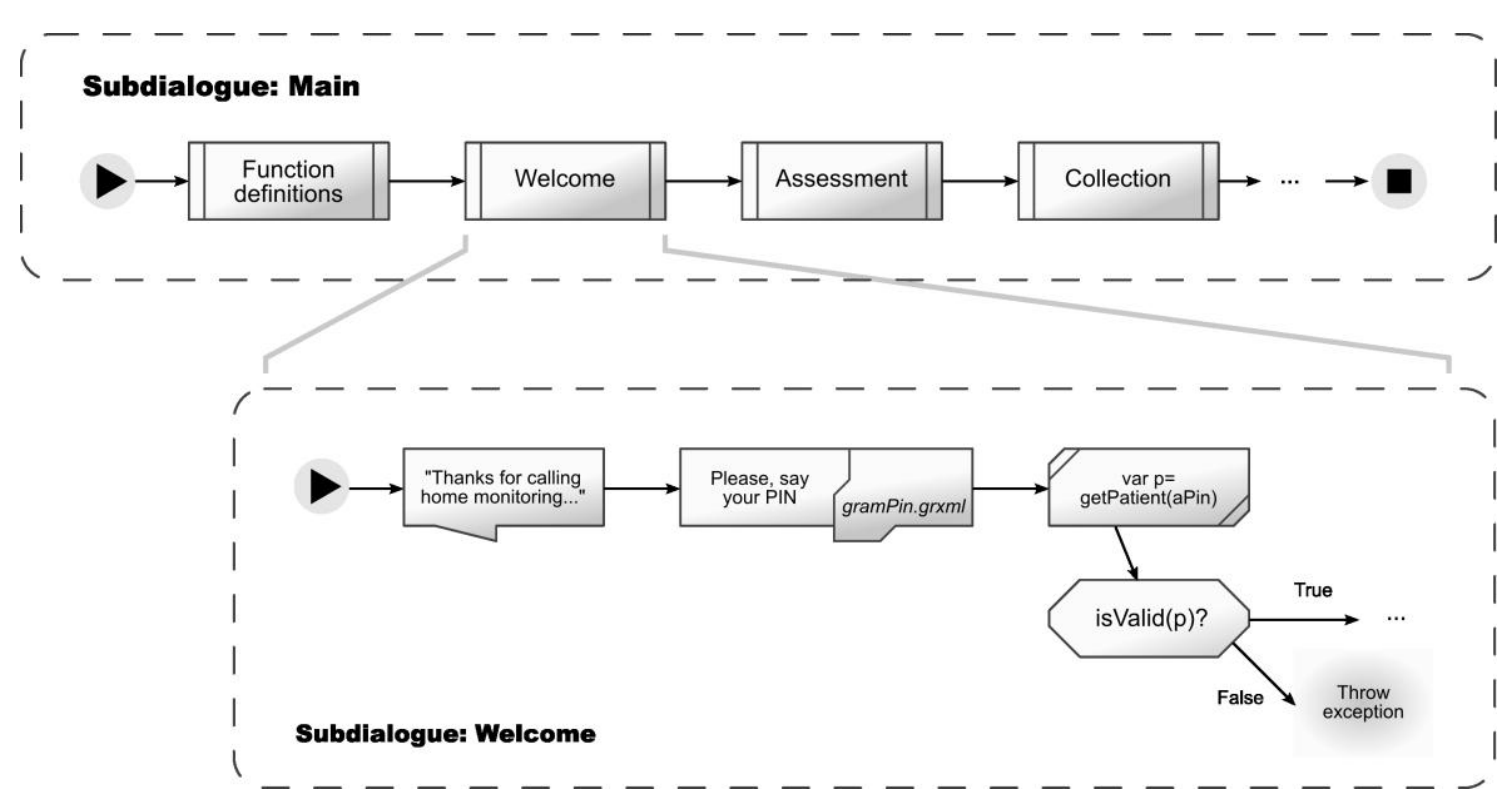

Figure 2 Block-based dialogue description. Subdialogues are defined by the application developer (shown here as rounded dotted boxes), and can be invoked with a subdialogue calling block (shown in double-border).

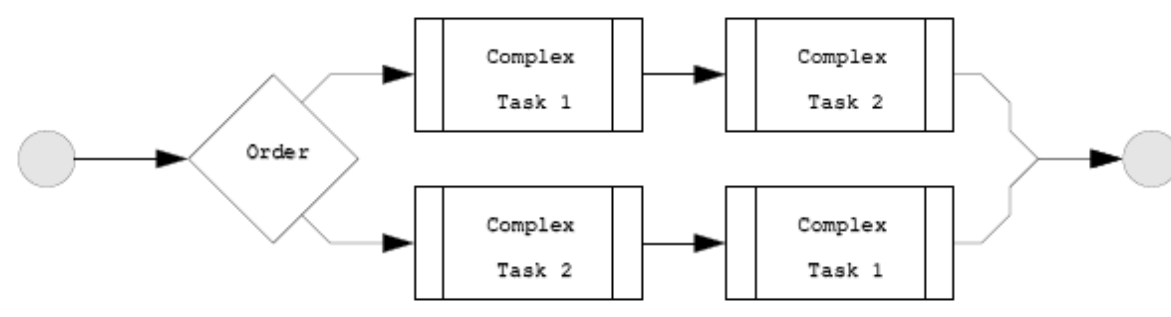

Figure 3 Within-application reuse of blocks. Dialogue sequence is rearranged without duplication

Several blocks are available for building subdialogues, namely: prompt, question, script, decision, exception handler, prompt sets, place-holders, containers and subdialogue calling blocks (Table I).

\begin{tabular}{ll}
\hline Block Class & Description \\
\hline Prompt & Evaluates its content and realizes the result in speech \\
Question & Evaluates and plays its content ; activates a grammar, and \\
& binds the return value to an object \\
Script & Allows bulk evaluation of arbitrary procedural and object- \\
& oriented code; may be used e.g. for defining functions, \\
\hline
\end{tabular}




\begin{tabular}{|c|c|}
\hline & $\begin{array}{l}\text { evaluating complex formulas, and accessing external } \\
\text { resources and libraries }\end{array}$ \\
\hline Decision & $\begin{array}{l}\text { If- and switch-like statements evaluate their arguments and } \\
\text { consequently alter the block execution flow }\end{array}$ \\
\hline Subdialogue & Activates a different subdialogue placing it on the call stack \\
\hline Exception handlers & $\begin{array}{l}\text { Exception handlers (catch) and generators (throw) handle } \\
\text { out-of-band events, including "no-match" and similar and } \\
\text { user-defined events. }\end{array}$ \\
\hline Container & $\begin{array}{l}\text { Multiple blocks can be grouped into a container, and } \\
\text { activated according to a chosen criterion - e.g. incrementally } \\
\text { across calls, to provide different information in subsequent } \\
\text { contacts; or according to any suitable "profile ability } \\
\text { function". }\end{array}$ \\
\hline Placeholder & $\begin{array}{l}\text { Can be instantiated as no-operation blocks, and their } \\
\text { implementation deferred to later }\end{array}$ \\
\hline Head & One-time declarations, e.g. directions for database access \\
\hline
\end{tabular}

Table I Main block classes for the AdaRTE dialog formalism

Containers are another important feature that supports building of natural-sounding interactions; they are used for common tasks in which one of several subdialogues are selected according to a specified policy (Figure 4). Containers provide a direct way for designers to augment the flexibility of the dialogue, for instance allowing prompts to accommodate to users' experience with the system. Policies for activations of blocks inside containers could be: randomly, in sequence, ordered by call number and according to an externally defined schedule. Another policy could be generated, for instance, by performing statistical analysis to classify the level of experience of the user or even the likelihood of their encountering problems on specific parts of the dialogue.

Equally central, along with the declarative block structure, is the embedded procedural interpreter. It provides an execution space which is shared with the block structure. Inclusion of procedural code is essential for flexibility, inter-operability, and ease of programming. Almost all prompts, questions and 
other user-visible elements are evaluated dynamically at run-time. Evaluation can include function and method calls; this allows, for example, to model entities (like, for examples, dates, therapy and doses) as complex objects. Therefore, they can be converted to their various natural-language representations via convenient calls to their methods. Furthermore, AdaRTE allows embedding larger blocks of code written in the ECMAScript language into script blocks. Procedural code has extensive access to external resources, Java standard libraries, and user-provided APIs. Note that, in contrast to VoiceXML, the script interpreter belongs to the server side, and thus it has access to external resources such as databases, ontologies, or any other commodity library.

Standard-conforming VBs provide basic mechanisms for semantic interpretation, e.g. through compliance to the SISR specification [35]. Grammars conforming to the specification construct objects and set their properties according to the parts of the sentence recognized. These objects are transferred to the AdaRTE server; in this way, semantic interpretation can happen both on the client, according to the SISR rules, or be coded in the server, e.g. using regular expressions, statistical text models, or NLP libraries.

Vendor-independence is an important consequence of the architecture of AdaRTE; it operates with offthe-shelf speech recognition software; in particular, for telephone interactions it acts as a web server and dynamically transmits VoiceXML code to a voice browser. The VB, which is in turn connected to other hardware, will be in charge of interpreting documents according to user's interaction over the phone. The browser captures and recognizes the answers, and sends them back to AdaRTE through HTTP requests. Vendor independence means that VBs can be replaced depending on economic considerations, quality of the recognition, languages supported, or even outsourced.

A large body of research is available on the optimization of spoken interfaces. Some of the results of the research have been condensed into best practices [36]. More complex confirmation strategies with respect to simple "yes/no" answers, for example, should be adapted according to confidence thresholds and $n$ best lists. The inclusion of such techniques into custom-developed systems is complex. A big advantage in using an interpretable and high-level dialogue representation language like the one proposed in this work is that such "dialogue practices" can be incorporated seamlessly into the underlying dialogue 
interpretation, removing the burden from the dialogue developer. Within the AdaRTE formalism, for example, developers can enable either plain or skip-list based confirmation steps to questions which are more prone to ASR misrecognitions (e.g. critical questions, or those activating more complex language models). These features will be handled internally to the server-side, which will map them into lowerlevel constructs.

A well-known limitation of conventional ASR grammars is that - on field - they do not perform well in some domains, e.g. with lists of drug names. Since AdaRTE is independent of the VB, more complex language models can be employed, as long as they are offered by the underlying platform. This includes, for example, especially-trained $n$-grams, or speaker-dependent adaptations.

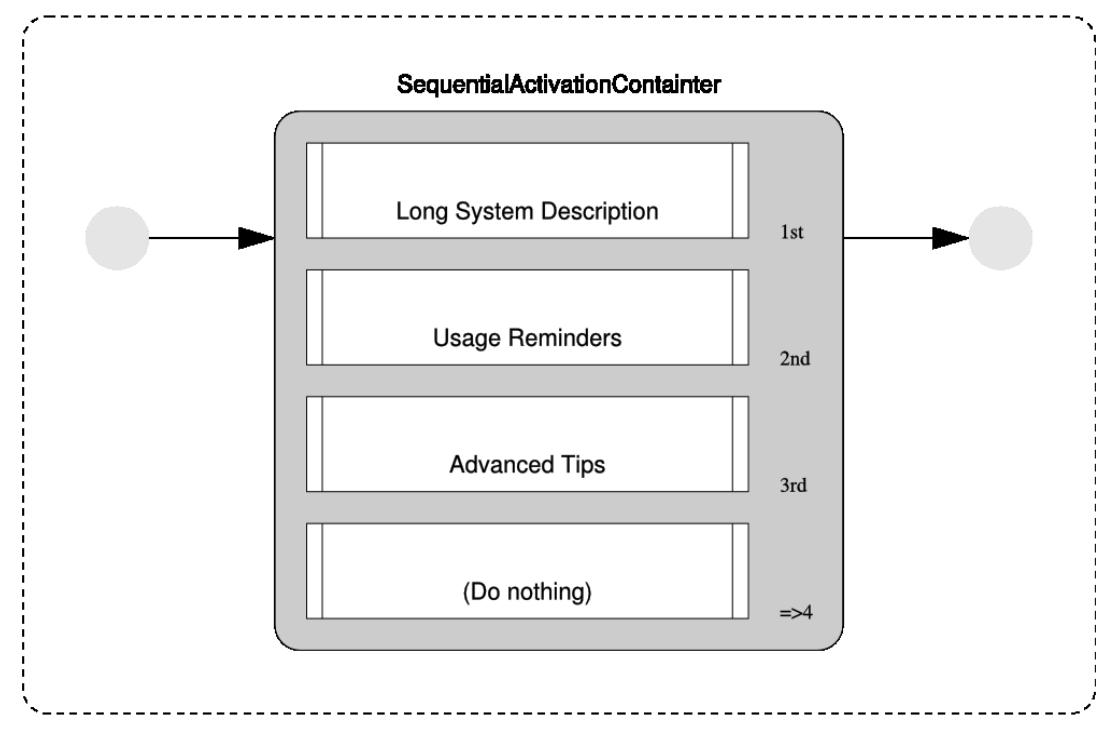

Figure 4 Containers automate switching between homologous blocks. Switching happens according to a container-specific policy - in this case, only one contained block is activated per invocation, according to call number for that patient: a long system description is played on the first call, a brief reminder is given on the second time he calls, and so on. Containers simplify the addition of variability to the dialogue.

\section{Evaluation and results}

The AdaRTE framework is currently fully operative and it has been integrated with three Voice Service Providers (VSPs). In this section we present the evaluation of the framework through the construction of three realistic health care dialogue systems derived by actual systems, which had been deployed and 
validated in the past, and two more test cases for peritoneal dialysis and OAT assistance. Implementation details are also presented in this section. A number of practical examples are displayed, describing the implementation of some of the important features explained in section 4.

\subsection{Evaluation}

AdaRTE was evaluated by constructing five health-care dialogues, paying special attention to the metrics that allow us to measure each dialogue development process. As a consequence, the strength of the framework is demonstrated by describing the variety of supported voice applications. At first, we consider as metrics the time invested, the expertise of developers and the technology and platform used in each developed dialogue, as shown in Table II. The first dialogue prototype is based on the TLC-COPD system previously deployed by the Friedman et al. [3]. For this specific example, we used Tellme Studio as VSP. The implementation of this pilot required less than two weeks of man-effort. The fulfilled activities, shown in Figure 5, included database schema definition, data preparation and dialogue deployment. This dialogue is in English language and based on phone keypad interaction (also known as touch-tones, based on dual-tone multi-frequency or DTMF).

\begin{tabular}{|c|c|c|c|c|c|c|c|}
\hline \multirow[b]{2}{*}{ No. } & \multirow[b]{2}{*}{ Prototype } & \multicolumn{2}{|c|}{ Development } & \multirow{2}{*}{\multicolumn{2}{|c|}{ - Mode Grammar }} & \multirow[b]{2}{*}{ Platform } & \multirow[b]{2}{*}{ Lang. Ref. } \\
\hline & & Time & Prof & & & & \\
\hline 1 & $\begin{array}{l}\text { Chronic Obstructive } \\
\text { Pulmonary Desease }\end{array}$ & 2 & Expert & DTMF & -- & $\begin{array}{l}\text { Tellme Studio, } \\
\text { Loquendo }\end{array}$ & English [3] \\
\hline 2 & $\begin{array}{l}\text { Hypertensive Patients } \\
\text { Management }\end{array}$ & 3 & Expert & Speech & $\begin{array}{l}\text { GSL } \\
\text { SGRS }\end{array}$ & $\begin{array}{l}\text { Voxpilot, } \\
\text { Loquendo }\end{array}$ & Italian [4] \\
\hline 3 & Diabetes & 4 & $\begin{array}{l}\text { Non- } \\
\text { Expert }\end{array}$ & Speech & SGRS & Loquendo & Italian [5] \\
\hline 4 & Dialysis & 4 & $\begin{array}{l}\text { Non- } \\
\text { Expert }\end{array}$ & Speech & SGRS & Loquendo & Italian \\
\hline 5 & $\begin{array}{c}\text { Oral Anticoagulation } \\
\text { Therapy }\end{array}$ & 4 & $\begin{array}{l}\text { Non- } \\
\text { Expert }\end{array}$ & Speech & SGRS & Loquendo & Italian -- \\
\hline
\end{tabular}

Table II - Five test-case health dialogues implemented in AdaRTE. The first three prototypes were based on previous implementations (see reference). GSL is the Nuance Grammar Specification Language; SRGS is the Speech Recognition Grammar Specification from the WWW Consortium[37]. 


\begin{tabular}{|c|c|c|c|c|c|c|c|c|c|c|c|c|c|c|c|c|c|c|c|c|c|c|c|}
\hline \multirow[b]{2}{*}{ Tasks } & \multicolumn{7}{|c|}{ Week 1} & \multicolumn{8}{|c|}{ Week 2} & \multicolumn{8}{|c|}{ Week 3} \\
\hline & $\mathrm{Su}$ & $M$ & $\bar{T}$ & W & Th & $\bar{F}$ & $\bar{S}$ & S & & $\bar{M}$ & $\bar{T}$ & $\bar{W}$ & Th & $\bar{F}$ & $\mathrm{~S}$ & & & $\bar{M}$ & $\mathrm{~T}$ & $\bar{W}$ & Th & $F$ & $\mathrm{Sa}$ \\
\hline $\begin{array}{l}\text { COPD Dialog analysis and db } \\
\text { schema definition }\end{array}$ & & & & & & ᄃ & & & & & & & & & & & & & & & & & \\
\hline COPD Deployment and testing & & & & & & & & & & & & & & & & & & & & $\sqsupset$ & & & \\
\hline
\end{tabular}

Figure 5 Gantt diagram of the COPD dialogue pilot prototype implemented in AdaRTE.

The second test case has been the partial re-implementation of the Homey dialogue system for the care of hypertension. It included an extensive Electronic Health Records (EHR) system with storage of personal data and profiles, in order to support dialogue adaptability. The original system has been used at two Italian hospitals for approximately two years [4]. Despite the successful deployment, the time spent in developing the original system (Figure 6) has been rather long and the result was reusable only to a limited extent. The voice part of the Homey system, for instance, took approximately one man-year for design and implementation. Re-engineering the system from the original proprietary dialogue manager to the AdaRTE architecture required approximately three weeks (eleven days of man effort). This valuable test case allowed a side-to-side comparison between different dialog development environments. The redevelopment of this prototype involved the following activities: VSP evaluation, database definition, and grammars and dialogue deployment (Figure 7). Unlike the TLC-COPD pilot, this system makes extensive use of grammars for speech input; grammars were formulated both in the GSL (Nuance 7) and the SRGS grammar formats [37], and the dialogue was tested by using the Voxpilot and Loquendo VoxNauta 7.0 VSP platforms. 


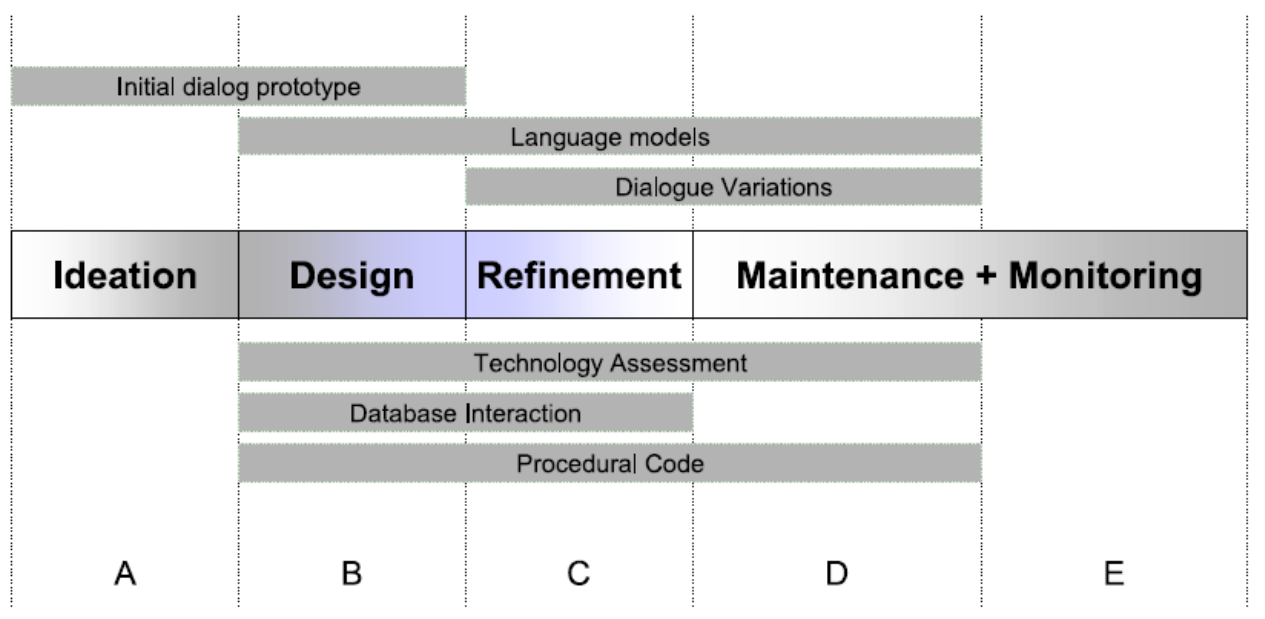

Figure 6 Phases in the development cycle of the original context-based Homey system.

\begin{tabular}{|c|c|c|c|c|c|c|c|c|c|c|c|c|c|c|c|c|c|c|c|c|c|}
\hline \multirow[b]{2}{*}{ Tasks } & \multicolumn{7}{|c|}{ Week 1} & \multicolumn{7}{|c|}{ Week 2} & \multicolumn{7}{|c|}{ Week 3} \\
\hline & $\mathrm{Su}$ & $\bar{M}$ & $T$ & W & Th & $\mathrm{F}$ & Sa & $\mathrm{Su}$ & $\bar{M}$ & $\mathrm{~T}$ & $\mathrm{~W}$ & Th & $\mathrm{F}$ & Sa & $\mathrm{Su}$ & $\bar{M}$ & $\mathrm{~T}$ & $\mathrm{~W}$ & Th & $\mathrm{F}$ & Sa \\
\hline $\begin{array}{l}\text { Voice Portal Service evaluation for } \\
\text { Italian Language }\end{array}$ & & & & & & & & & & 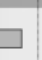 & & & & & & & & & & & \\
\hline $\begin{array}{l}\text { HOMEY Dialog analysis and db } \\
\text { schema definition }\end{array}$ & & $\square$ & & & & ב & & & & & & & & & & & & & & & \\
\hline Grammars deployment & & & & & & & & & & $\square$ & & $\sqsupset$ & & & & & & & & & \\
\hline Dialog deployment and testing & & & & & & & & & ㄷ & & & & & & & $\sqsupset$ & & & & & \\
\hline
\end{tabular}

Figure 7 Gantt diagram of the Homey pilot developed in AdaRTE.

Dialogues 3-5 in Table II have been implemented by external, junior developers; all of them support speech input, are executed in Italian using Loquendo VoxNauta 7.0 as VSP. The third dialogue is based on the IVR used in the multi-access service for the management of diabetes mellitus patients (M2DM) project [5]. The goal of this dialogue is to enquire information regarding insulin and glucose selfmeasurements by diabetic patients. The dialogue adapts its interaction according to patient's therapy. The fourth dialogue provides assistance to patients undergoing peritoneal dialysis. It interacts to obtain information concerning the home dialysis process and the health status. The dialogue adapts its interaction according to patient's expertise, answers and history as shown in Figure 8. The expertise level is calculated on the average of "no match" and "no input" events registered during a call. The patient's history gathers the clinical information, the answers on previous calls and the typology of the dialysis therapy. 


\section{Call No. 1}

1) Did you have difficulties in breathing?

2) Did you have cloudy liquid discharge?

3) Have you ever suffered from catheter pain?

Call No. 2

1) Did you have further difficulties in breathing?

2) Did you continue suffering from catheter pain?

Figure 8 Sample of adapting questions across calls for the tele-dialysis dialogue prototype.

The last dialogue was designed to communicate the daily therapy to patients under OAT, and to verify that the patient has understood correctly the therapy. This dialogue is being designed with the Mondino Neurological Hospital of Pavia (Italy).

Other metrics were used to measure the completeness and usability of AdaRTE, demonstrating that the framework covers all of the features previously introduced in Section 4 and indicating the level of complexity of the dialogues that can be implemented in the framework. Conceptually, the metrics were classified in database complexity (D), language-model complexity (L), application-complexity (C), and front-end complexity (F) as shown under the column "type" of Table III. For instance, the EHR designed for the Homey system has 78 tables and it is only accessed 6 times during the dialogue. The explanation for this fact is that the Homey database (like the OAT) is a shared resource, accessed by other interfaces besides the vocal one. In order to evaluate the complexity of the language model, the internal representation of generated and recognized speech were considered. The former indicates whether or not the dialogue exploits the SISR object-based mechanisms for the semantic representation of recognition results, while the latter describes whether or not the dialogue uses an object-oriented approach to generate the output messages. Grouping prompts into objects turned out to be an especially effective programming technique: often the same utterance should be conveyed in several styles with slight linguistic variations (brief or verbose, singular or plural, request to repeat, etc.). Representing the utterance with the instance of an object allows the programmer to add more variations as the need arises, still handling the object as a unit. 
The number of custom grammars conveys an idea of the domain-specific grammars implemented in each dialogue. It is important to mention that many built-in grammars, offered by the VB e.g. numbers, boolean, etc., were adopted and other custom grammars were re-used in dialogues.

The number of code lines of the whole dialogue, the length of the embedded scripts, and the number of blocks provide an estimate of the level of complexity of each dialogue application. Of course, the estimate is just a rough indication, because the dialogue code can usually be simplified by expert developers and, on the other hand, expanded by liberal use of comments. The number of ECMAScript code lines indicates how much the implemented dialogues took advantage of the benefits of the dynamic procedural interpreter; such code is mainly used for external resources access and decision making.

Table IV compares the code-line counts for the previous proprietary context-based implementation of the Homey system with the AdaRTE-based reengineering. Despite being essentially the same application, a large amount of procedural code was formerly required for a relatively small number of database accesses, which retrieved the patient's profile at the beginning of the dialog and stored call outcomes at its conclusion. This was mainly due to the fact that database access mechanisms were not part of the dialog primitives. Also, lots of code were required for user profiling, because the simple procedural interpreter was somewhat unsuited for the evaluation of the complex criteria required for adaptability (e.g. decision making to alter the dialog flow with respect to user ability, checking ranges with respect to previous readings, and so on).

Table III depicts another metric, i.e. the number of blocks adopted by each dialogue, grouped by block type. All the implemented dialogues used questions, prompts, scripts and exception handlers' blocks. Not surprisingly, information-gathering and patient-monitoring applications (dialogues 1-4) have a larger fraction of question blocks with respect to the OAT dialog, which is primarily an information-providing application, in which prompt blocks are prominent. The no-match and no-input are Exception blocks, relevant for controlling these VB exceptions.

\begin{tabular}{clccccc}
\hline Type & \multicolumn{1}{c}{ Metrics } & COPD & Homey & Diabetes & Dialysis & OAT \\
\hline D & Number of DB access & 19 & 6 & 26 & 13 & 10 \\
D & $\begin{array}{l}\text { Number of DB tables } \\
\text { Internal representation of }\end{array}$ & 12 & 78 & 17 & 31 & 12 \\
L & speech/semantics & No & Yes & Yes & Yes & Yes
\end{tabular}


Internal representation of

L generated speech

L Number of custom grammars

$$
\text { No }
$$

0

Total code lines

No
13

C Number of subdialogues

Questions

781

15

No

No Yes

Prompts

Scripts

Number of Exception

C blocks

handler

Containers

No-match/

No-Input

C Ecmascript code lines

F Modality (Voice/DTMF)

F N-best/skip-list adoption

Table III The metrics used to represent the complexity of the developed dialogues were grouped into: $D$ = Database Complexity, $L=$ Language Model Complexity, $C=$ Application complexity and F=Front-end complexity.

\begin{tabular}{|c|c|c|c|}
\hline Type & Metrics & $\begin{array}{l}\text { Homey: } \\
\text { Custom }\end{array}$ & $\begin{array}{l}\text { Homey: } \\
\text { AdaRTE }\end{array}$ \\
\hline $\mathrm{D}$ & Number of DB access & 2 & 6 \\
\hline $\mathrm{D}$ & Number of DB tables & 78 & 78 \\
\hline $\mathrm{L}$ & $\begin{array}{l}\text { speech/semantics } \\
\text { Internal representation of }\end{array}$ & Yes & Yes \\
\hline $\mathrm{L}$ & $\begin{array}{l}\text { Internal representation of } \\
\text { generated speech }\end{array}$ & No & No \\
\hline $\mathrm{L}$ & Number of custom grammars & $\sim 100$ & 13 \\
\hline $\mathrm{C}$ & Total code lines & 10132 & 781 \\
\hline \multirow[t]{5}{*}{$\mathrm{C}$} & Number of subdialogues & 26 & 21 \\
\hline & Questions & - & 33 \\
\hline & Prompts & - & 11 \\
\hline & Scripts & - & 14 \\
\hline & Exception & - & \\
\hline \multirow[t]{4}{*}{$\mathrm{C}$} & handler & & 8 \\
\hline & Containers & - & 1 \\
\hline & No-match/ & - & \\
\hline & No-Input & & 6 \\
\hline $\mathrm{C}$ & Procedural code lines & 8776 & 396 \\
\hline $\mathrm{F}$ & Modality (Voice/DTMF) & Voice & Voice \\
\hline $\mathrm{F}$ & N-best/skip-list adoption & No & Yes \\
\hline
\end{tabular}

Table IV A comparison between the custom context-based Homey dialog and the AdaRTE-based prototype reimplementation.

The front-end complexity was measured considering the modality and the adoption of the confidence thresholds and n-best lists. The HOMEY dialogue, for example, activated the $n$-best confirmation strategy inside question blocks. Thus, in case of ASR misrecognition, the utterance will be added into the skip-list 
that contains elements to be discarded by the ASR in future recognitions during the whole confirmation process of the question under discussion.

\subsection{Examples}

Practical examples of the implementation of dialogues are presented in Figures 9 to 12. Figure 9 displays the "root" subdialogue, main, of the COPD dialogue description. Note that it invokes the main topics to be addressed in the dialogue by subdialogue call blocks. Figure 10 presents the hierarchical structure of the subdialogues that make up the Dialysis dialogue and their flow. Figure 11 and 12 provide examples of script blocks; they define functions that retrieve some patient's data from the database. Figure 11 shows a basic method for placing a query: a structured query language (SQL) query statement is constructed and delivered directly to the database via the Java Database Connectivity (JDBC) API [38]. In contrast, Figure 12 shows a more sophisticated approach adopted in the OAT dialogue, which uses the Hibernate persistence framework to access the databases [39]. A persistence framework abstracts the mechanics of the query language, binding database entities to programming language objects.

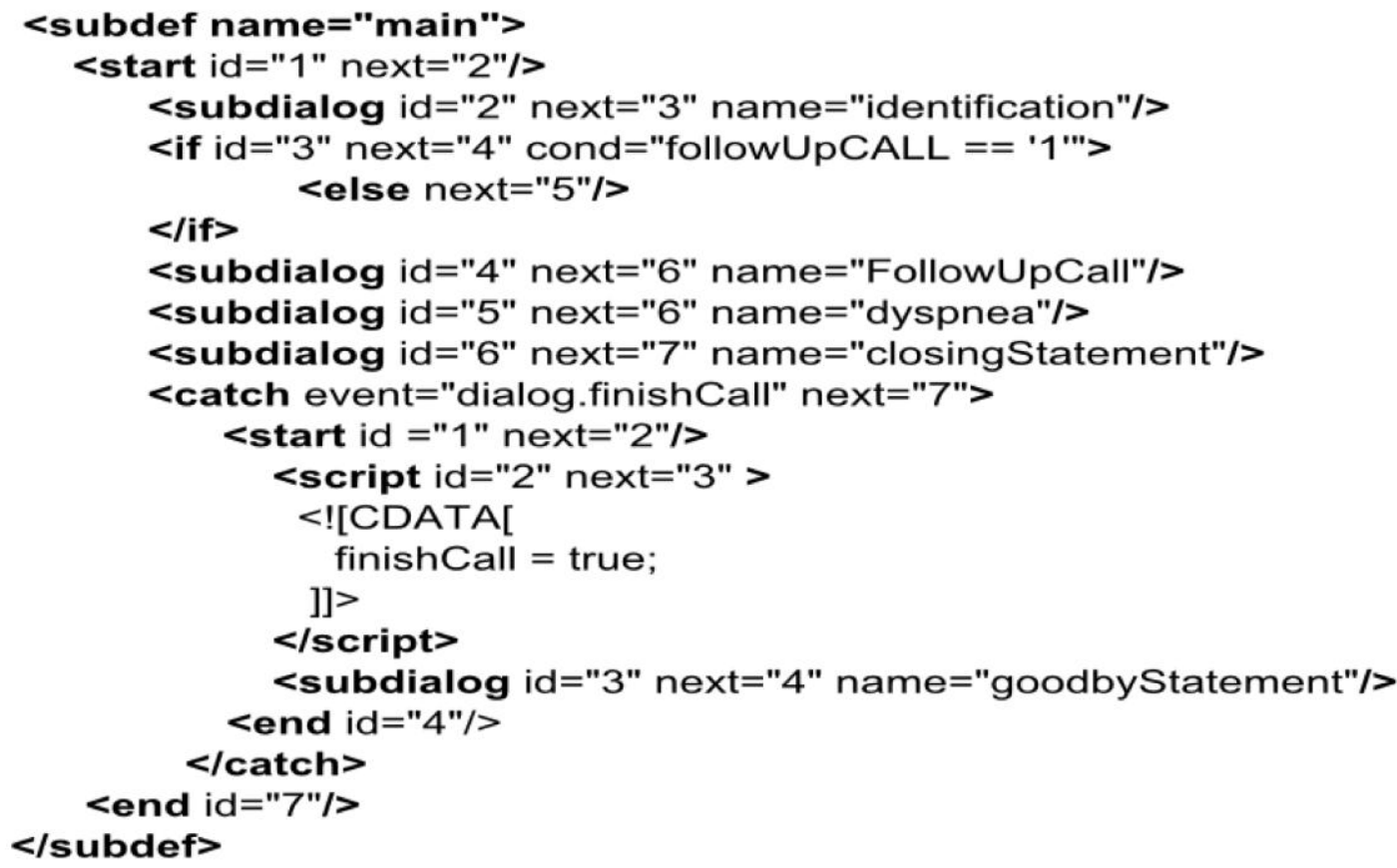

Figure 9 Top-level dialog sequence (COPD example). 


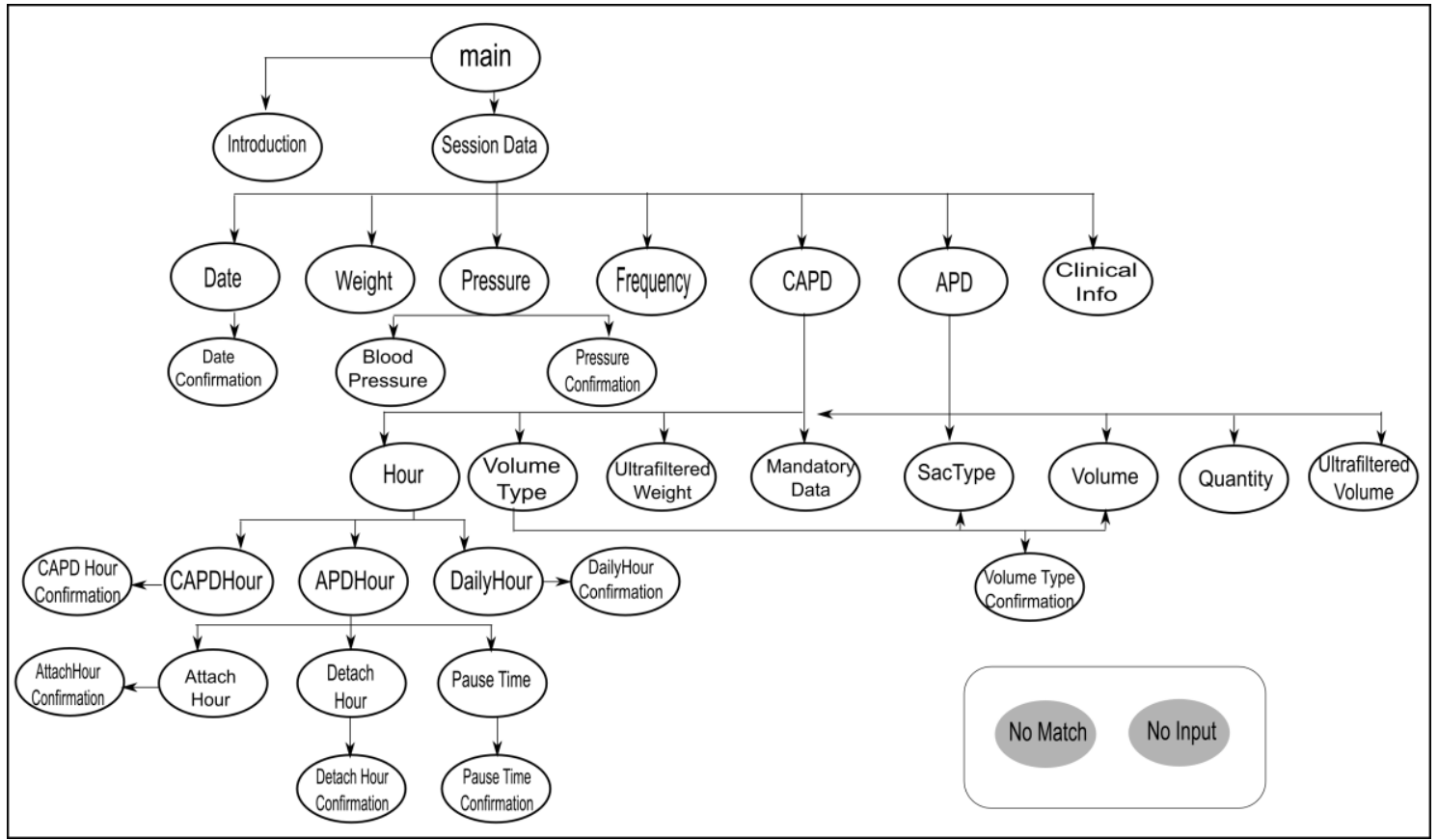

Figure 10 Hierarchical structure of subdialogues in the dialysis vocal application.

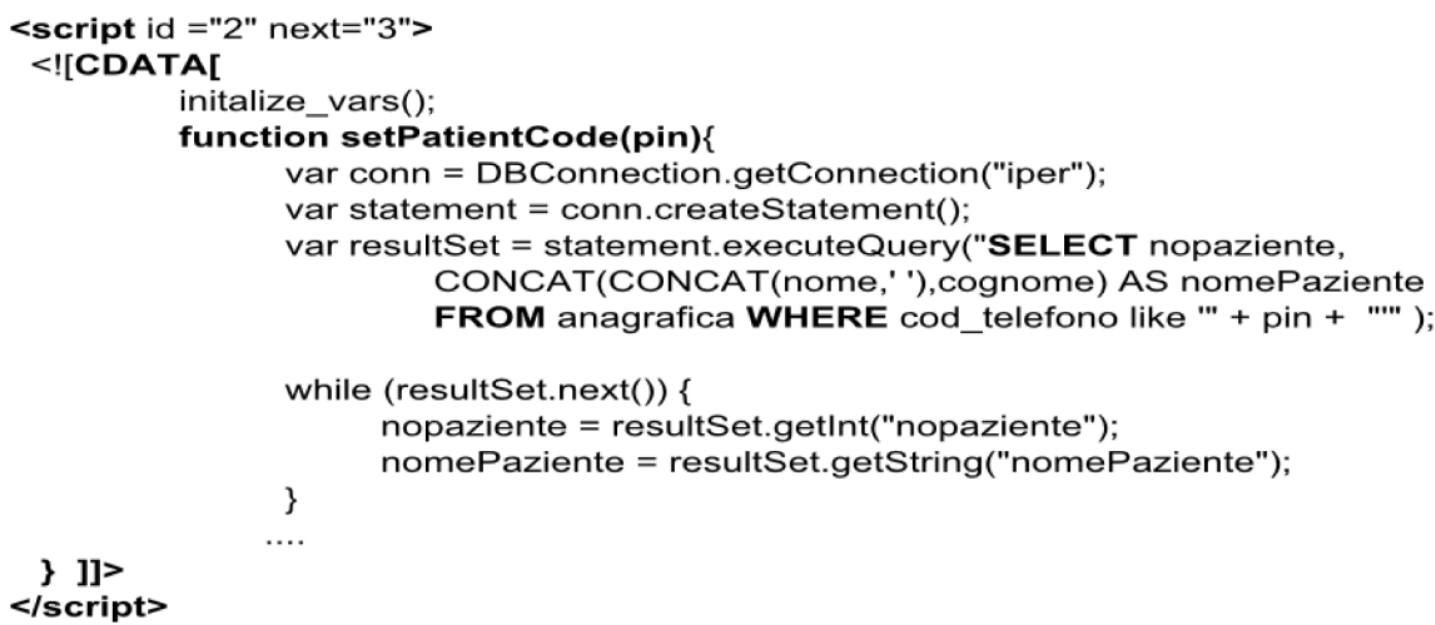

Figure 11Procedural code of a script block that access a database through the JDBC API (authentication). 


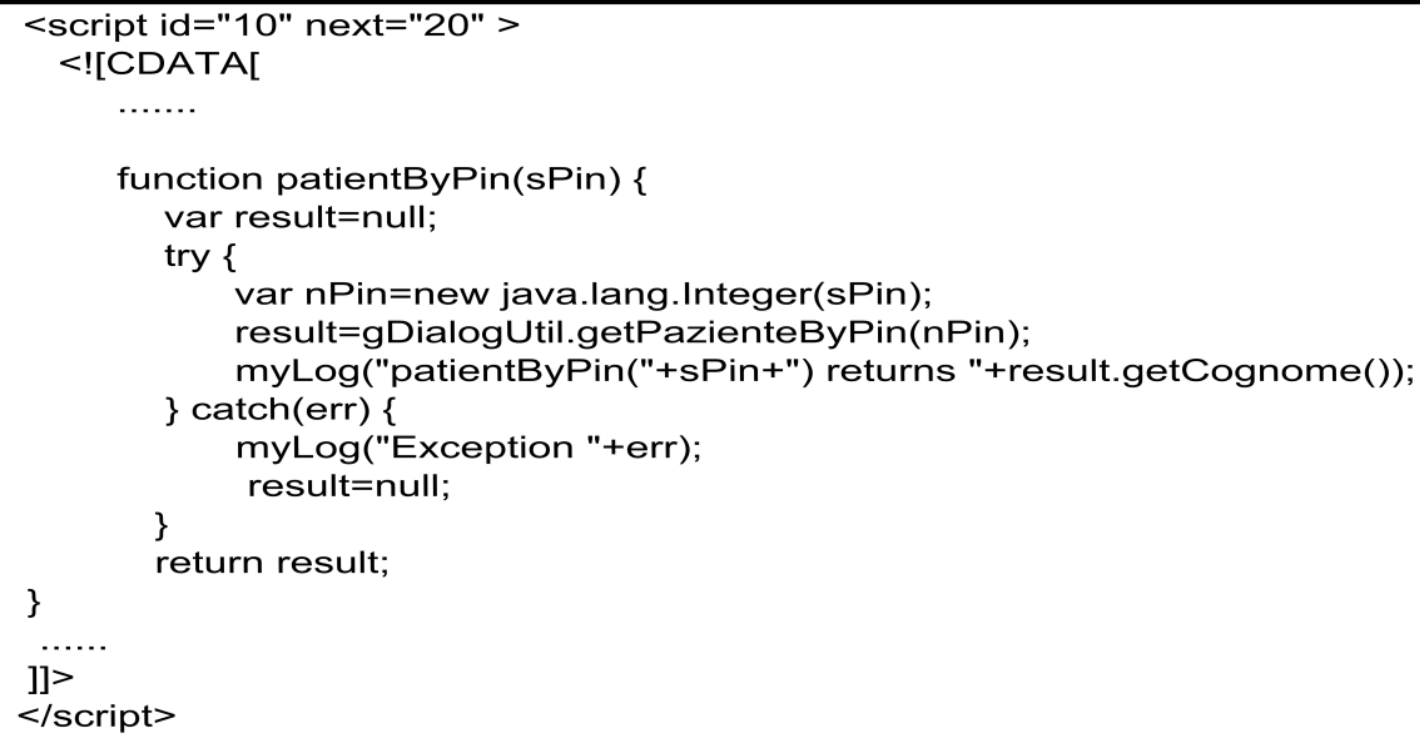

Figure 12 Procedural code in a script block of the OAT dialogue. This code retrieves an object representing the patient ("result"), given their personal identification number ("pin"). The actual work is performed by a method of the Java commodity object "gDialogUtil", which accesses the database by means of the Hibernate persistence framework.

\section{Discussion}

We presented five health-care dialogues that have been implemented in AdaRTE. Whilst implementing these applications, developers profited from the features for rapid prototyping reuse, adaptable decision support and best-practices provided by the framework. Through these dialogues it has been proved that the framework fully supports these features. Moreover, the completeness and usability of the framework has been measured by describing the complexity of realistic applications that could be implemented with it.

In conclusion, the research and development effort provided an operative solution for rapid prototyping of health dialogues, with a level of functionality that is not attained by current frameworks supporting more complex theoretical approaches to dialogue. These frameworks should consider not only the intrinsic complexity of dialogue modelling, but also the special requests in the medical domain, in order to offer a leaner development of robust and efficient dialogues. This is an important direction for future research in computational linguistics and medical informatics. 


\section{Future enhancements}

Currently, we have a strong commitment on the integration of a more elaborated semantic interpretation by integrating AdaRTE with an NLP application that supports lexicalized grammars to increase expressivity [40]. In this way, not only recognition would not depend on the grammars supported by VBs, but also more natural interactions will be supported improving the patient's perception of dialogues.

Inclusion of spoken interfaces optimization techniques or best practices into custom-developed systems is not straightforward. A big advantage in using an interpretable and high-level dialogue representation language like the one proposed in this work is that more "dialogue practices" can be incorporated seamlessly into the underlying dialogue interpretation, removing the burden from the dialogue developer.

Furthermore, extended support to the management of voice projects is foreseen, where a project involves a dialogue and its composing subdialogues, together with definitions of templates. High level templates serve as guidelines in the development of abstract tasks, e.g. for assessing the patient's psychological stage (useful e.g. for behaviour-change interventions based on psychologically-motivated models).

The AdaRTE system was foreseen not only as a reliable platform for dialogue deployment, but also as a framework for incorporating advanced features of speech recognizers as they become available, including increased support to adaptability, and natural language understanding and generation. For instance, so far AdaRTE supports the same amount of mixed initiative provided by the underlying VoiceXML interpreter. This could be enhanced by introducing stochastic-based grammars in the VSPs in order to increment the variety of possible expressions and the specialized medical terminology. Similarly, a more elaborated semantics representation could be considered in future frameworks for easy deployment of dialogues in health. 


\section{Conclusion}

We have presented a dialogue-interpretation architecture for rapid dialog prototyping. The corresponding engine addresses current barriers to the realization of elaborate telephone-based interactions. AdaRTE differs significantly from other frameworks because it is targeted at the requirements of the chronic-care domain, which typically requires adaptable dialogs with complex structures and enquiry data collection tasks. The new methodology offers developers a high level of flexibility, by allowing dynamically access through the procedural execution environment surrounding the interpreter. At the same time, dialogs can be coded and inspected by developers which are not specifically trained in web-based technologies.

The expressiveness of the dialogue representation yielded an important reduction of the time invested in developing a number of real-world prototypes. We have implemented five health-care dialogue prototypes and showed that development times were remarkably optimized with respect to earlier development methodologies. Finally, AdaRTE is a standard-compliant architecture for the incremental adoption and experimentation with advanced dialog formalisms.

\section{Acknowledgments}

The authors would like to thank Prof. R. Friedman (Medical Information Systems Unit, Boston, MA) for information on the TLC-COPD dialogues; Prof. T. Bickmore (College of Computer and Information Science, Northeastern University, Boston, MA) for the discussions that led to the AdaRTE project; Dr. A. Cavallini (IRCCS Mondino, Pavia) for the OAT project; I. Buetti, I. Limongelli, F. Carpignano, S. Derosa, and others for contributing to dialogues test cases and for the useful discussions.

\section{References}

1. Bickmore T, Giorgino T. Health dialog systems for patients and consumers. Journal of biomedical informatics. 2006;39(5):556-71.

2. Lenz R, Blaser R, Beyer M, Heger O, Biber C, Bäumlein M, et al. IT support for clinical pathways-Lessons learned. International Journal of Medical Informatics Elsevier. 2007;76:S397-S402 3. Young M, Sparrow D, Gottlieb D, Selim A, Friedman RH. A telephone-linked computer system for COPD care. Chest. 2001;119:1565-75. 
4. Giorgino T, Azzini I, Rognoni C, Quaglini S, Stefanelli M, Gretter R, et al. Automated spoken dialogue system for hypertensive patient home management. International Journal of Medical Informatics Elsevier. 2004;74:159-67.

5. Larizza C, Bellazzi R, Stefanelli M, Ferrari P, De Cata P, Gazzaruso C, et al. The M2DM Project The Experience of Two Italian Clinical Sites with Clinical Evaluation of a Multi-access Service for the Management of Diabetes Mellitus Patients. Methods of Information in Medicine. 2006;45:79-84.

6. Piette JD. Interactive voice response systems in the diagnosis and management of chronic disease. Am J Manag Care. 2000;6:817-27.

7. Corkrey R, Parkinson L. Interactive voice response: review of studies 1989-2000. Behav Res Methods Instrum Comput. 2002;34:342-53.

8. Krishna S, Balas EA, Boren SA, Maglaveras N. Patient acceptance of educational voice messages: a review of controlled clinical studies. Methods Inf Med. 2002;41:360-9.

9. Kaplan B, Farzanfar R, Friedman RH. Personal relationships with an intelligent interactive telephone health behavior advisor system: a multimethod study using surveys and ethnographic interviews. International Journal of Medical Informatics Elsevier. 2003;71(1):33-41.

10. Edmonds M, Bauer M, Osborn S, Lutfiyya H, Mahon J, Doig G, et al. Using the vista 350 telephone to communicate the results of home monitoring of diabetes mellitus to a central database and to provide feedback. International Journal of Medical Informatics Elsevier. 1998;51(2-3):117-25.

11. Migneault JP, Farzanfar R, Wright JA, Friedman RH. How to write health dialog for a talking computer. Journal of biomedical informatics. 2006 Oct;39(5):468-81.

12. Levin E, Levin A. Evaluation of Spoken Dialogue Technology for Real-Time Health Data Collection. J Med Internet Res. 2006;8(4):e30.

13. Bickmore T, Giorgino T, Picard R. Guest Editorial: Special Issue on Dialog Systems for Health Communication. Journal of Biomedical Informatics Elsevier. 2006;39:465-7.

14. Zafar A, Mamlin B, Perkins S, Belsito AM, Overhage JM, McDonald CJ. A simple error classification system for understanding sources of error in automatic speech recognition and human transcription. International Journal of Medical Informatics Elsevier. 2004;9-10:719-30.

15. Allen J, Ferguson G, Blaylock N, Byron D, Chambers N, Dzikovska M, et al. Chester: Towards a personal medication advisor. Journal of biomedical informatics. 2006;39(5):500-13.

16. Beveridge M, Fox J. Automatic generation of spoken dialogue from medical plans and ontologies. Journal of biomedical informatics. 2006;39(5):482-99.

17. Allen J, Ferguson G, Stent A. An architecture for more realistic conversational systems. In Proceedings of Intelligent User Interfaces (IUI-01); 2001.

18. Power R. The organisation of purposeful dialogues. Linguistics An International Review La Haye 1979;17(1-2):107-51.

19. Allen J, Byron D, M. D, Ferguson G, Galescu L. Towards Conversational Human-Computer Interaction. AI Mag. 2001;22(4):27-37.

20. Perrault CR, Allen JF. A Plan-Based Analysis of Indirect Speech Acts. American Journal of Computational Linguistics. 1980;6(3-4):167-82.

21. Churcher G, Atwell, E., Souter, C. Dialogue management systems: a survey and overview. School of Computer Studies. University of Leeds.; 1997.

22. Larsson S, Traum D. Information state and dialogue management in the TRINDI dialogue move engine toolkit. Natural Language Engineering Special issue on spoken language dialogue system engineering. 2000;6:323-40.

23. McGlashan S, Burnett DC, Carter J, Danielsen P, Ferrans J, Hunt A, et al. Voice Extensible Markup Language (VoiceXML) Version 2.0.; 2004 [updated 2004; cited]; Available from: http://www.w3.org/TR/voicexml20/.

24. Black LA, McTear M, Black N, Harper R, Lemon M. Di@log by design: An Integrated TeleCare Communication Infrastructure. In: Proceedings of the 26th Annual International Conference of the IEEE EMBS San Francisco 2004;2:3290-3.

25. Mittendorfer M, Niklfeld G, Winiwarter W. Evaluation of Intelligent Component Technologies for VoiceXML Applications; 2001.

26. Mittendorfer M, Niklfeld G, Winiwarter W. Making the voice web smarter-integrating intelligent component technologies and VoiceXML. In: Proceedings of the Second International Conference on Web Information Systems Engineering 2002;2:126-31.

27. Falavigna D, Gretter R and Orlandi M. A mixed language model for a dialogue system over the telephone. ICSLP 2000; 2000; Beijing, China. 
28. Falavigna D, Giorgino T, Gretter R. A frame based spoken dialog system for home care. INTERSPEECH-2005; 2005.

29. Ecma International. Standard ECMA-262: ECMAScript Language Specification, 3rd edition.; 1999 December.

30. Hataoka N, Obuchi Y, Mitamura T, Nyberg E. Robust speech dialog interface for car telematics service. In: First IEEE Consumer Communications and Networking Conference 2004:331 - 5.

31. Hartman J, Vila JA. VoiceXML Builder: A workbench for investigation voice-based applications. In: Proceedings of the 31st Annual Frontiers in Education Conference, 2001 2001;3:S2C 69.

32. Hamerich S, Schubert V, Schless V, Crdoba R, Pardo J, d'Haro L, et al. Semi-Automatic Generation of Dialogue Applications in the GEMINI Project. Sigdial; 2004. 2004.

33. Di Fabbrizio G, Lewis C. Florence: a Dialogue Manager Framework for Spoken Dialogue Systems. 8th International Conference on Spoken Language Processing; 2004; Jeju, Jeju Island, Korea.

34. Balentine B, Morgan DP. How to Build a Speech Recognition Application. A Style Guide for Telephony Dialogues. Second ed.: EIG Press; 2001.

35. Van Tichelen L, Burke D. Semantic Interpretation for Speech Recognition (SISR) Version 1.0.: W3C Recommendation 5. , April 2007.

36. Weinschenk S, Barker T. Designing Effective Speech Interfaces. Wiley; 2000.

37. McGlashan S, Hunt A. Speech Recognition Grammar Specification Version 1.0. 2004 [updated 2004; cited]; Available from: http://www.w3.org/TR/speech-grammar/.

38. Hamilton G, Cattell R, Fisher M. Jdbc Database Access With Java: A Tutorial and Annotated Reference (Java Series). Addison-Wesley; 1997.

39. Bauer C. Java Persistence with Hibernate. Manning Publications; 2006.

40. Rojas-Barahona LM. Adapting Combinatory Categorial Grammars in a Framework for Health Care Dialogue Systems. Workshop on the Semantics and Pragmatics of Dialogue; 2007; Rovereto Italia. Edited by Ron Artstein and Laure Vieu.

\section{Address for correspondence}

E-mail address: linamaria.rojas@unipv.it (L. M. Rojas-Barahona). 